\title{
A!
}

This is an electronic reprint of the original article.

This reprint may differ from the original in pagination and typographic detail.

Puska, M. J.; Corbel, C.; Nieminen, R. M.

\section{Positron trapping in semiconductors}

Published in:

Physical Review B

DOI:

10.1103/PhysRevB.41.9980

Published: 15/05/1990

Document Version

Publisher's PDF, also known as Version of record

Please cite the original version:

Puska, M. J., Corbel, C., \& Nieminen, R. M. (1990). Positron trapping in semiconductors. Physical Review B, 41(14), 9980-9993. https://doi.org/10.1103/PhysRevB.41.9980

This material is protected by copyright and other intellectual property rights, and duplication or sale of all or part of any of the repository collections is not permitted, except that material may be duplicated by you for your research use or educational purposes in electronic or print form. You must obtain permission for any other use. Electronic or print copies may not be offered, whether for sale or otherwise to anyone who is not an authorised user. 


\title{
Positron trapping in semiconductors
}

\author{
M. J. Puska, C. Corbel, ${ }^{*}$ and R. M. Nieminen \\ Laboratory of Physics, Helsinki University of Technology, SF-02150 Espoo 15, Finland
}

(Received 20 October 1989)

\begin{abstract}
Positron trapping into vacancies in semiconductors is studied on the basis of Fermi's golden-rule calculations. The emphasis is put on the comparison of the trapping properties into defects in different charge states. In particular, the temperature dependences are investigated. Important features for vacancy-type defects in semiconductors are the localized electron states within the forbidden energy gap and (in the case of negatively charged defects) the weakly bound Rydberg states for positrons. Compared to vacancy-type defects in metals, these features make possible new kinds of trapping mechanisms with electron-hole and phonon excitations. For charged defects the Coulomb wave character of the delocalized positron states before trapping determines the amplitude of the wave function at the defect and thereby strongly affects the magnitude of the trapping rate. As a result, trapping into positively charged defects is effectively forbidden while negatively charged defects show remarkable properties which differ from the picture established for positron trapping in metals. The trapping rate into negative defects increases strongly with decreasing temperature and at very low temperatures "gigantic" values may result.
\end{abstract}

\section{INTRODUCTION}

Studies of defects in semiconductors by positron annihilation techniques have increased rapidly during the last years. ${ }^{1,2}$ Positron lifetime measurements have given new information about various properties of native and introduced defects. Theoretical work has had an important role in deepening the understanding of the positrondefect interactions in semiconductors. ${ }^{3-5}$ One of the key questions is how a positron makes a transition from a delocalized bulk state in the crystal to a localized state provided by a vacancy-like defect. This process is called "positron trapping." The most important issues in trapping are the capture mechanisms which determine the trapping coefficient, i.e., the magnitude of the transition rate, and its temperature dependence. Positron trapping has been much less studied in semiconductors than in metals where the subject is well documented both theoretically and experimentally. ${ }^{6,7}$ In semiconductors, there is experimental evidence that the characteristics of positron trapping into vacancy defects vary considerably between different semiconductors and with the position of the Fermi level in a given semiconductor. ${ }^{8-11}$ Positron capture at negative ions has also been observed. ${ }^{12}$ Different capture mechanisms have been discussed to explain the positron behavior but the present paper is the first one to report on a systematic theoretical survey and to present calculations of the resulting trapping coefficients.

The process of positron trapping into defects in metals is more or less well understood. ${ }^{6,7}$ In the case of spatially small traps such as vacancies the trapping process is transition limited. ${ }^{13}$ The trapping coefficient is a temperature independent constant of magnitude $\sim 10^{15} \mathrm{~s}^{-1}$. The dominant process taking care of the energy transfer from the positron to the host is electron-hole pair creation. Phonon creation is usually not important because the binding energy liberated is so large that it cannot be taken by a single phonon and multiphonon processes are very ineffective. In the case of larger voids the trapping process eventually becomes diffusion limited, and the trapping coefficient can depend on temperature. ${ }^{14}$ There have also been numerous suggestions of shallow traps (such as dislocations) which are operative only at low temperatures where thermal detrapping is slow and/or act as precursors to deeper traps (such as jogs in the case of dislocations). ${ }^{15}$ It has also been suggested that lowenergy scattering resonances could strongly enhance the trapping rate of epithermal positrons into small vacancy agglomerates. ${ }^{16,17}$

The knowledge of positron trapping in metals cannot be directly transferred from metals to semiconductors. The existence of the band gap modifies the densities of initial and final electron states in the electron-hole creation process. According to theoretical calculations ${ }^{3,4}$ the positron binding energy at a vacancy can often be smaller than the electron band gap so that electron transfer from the valence band to the conduction band becomes impossible. On the other hand, there are localized electron states in the band gap induced by the defects themselves. The excitation of electrons from these localized levels can provide an efficient channel for consuming the positron binding energy. In addition, electronic screening is reduced in semiconductors in comparison with that in metals. Thus the net positron-electron interaction is stronger in semiconductors, which has an enhancing effect for trapping.

Another important difference between metals and semiconductors is that defects in semiconductors may exist in several charge states. Positive defects are expected to repel positrons. Negatively charged defects are expected to be strongly attractive to positrons due to their attractive long-range Coulomb potential. This Coulomb tail can affect the capture mechanism into vacancies by 
inducing several positron Rydberg states with low binding energies. These states may act as precursor states in the trapping process and thereby enhance the overall trapping coefficient for the defect.

According to several measurements ${ }^{8-10}$ the positron trapping rate in semiconductors may in some cases depend strongly on temperature: when temperature decreases the trapping rate increases rapidly. For example, in a recent work Mäkinen et al. ${ }^{10}$ reported that the trapping rate into vacancies in $\mathrm{Si}$ increases by a factor of 10 when temperature decreases from 120 to $20 \mathrm{~K}$. On the other hand, in the case of heavily P-doped Si Mäkinen et al. found that the positron trapping rate is temperature independent as in metals. There exist only a few estimations for the trapping coefficient in semiconductors because the concentration of defects is rather difficult to estimate in most cases. The room-temperature trapping coefficient for divacancies in $\mathrm{Si}$ has been found to be similar to those in metals, i.e., $(2-3) \times 10^{14} \mathrm{~s}^{-1} .18,9$ The values estimated for As vacancies in GaAs and vacancy-P pairs in heavily $\mathbf{P}$-doped $\mathrm{Si}$ are slightly larger: $6 \times 10^{14}$ $\mathrm{s}^{-1}$ (Ref. 19) and $2 \times 10^{15} \mathrm{~s}^{-1},{ }^{10}$ respectively. In the case of the strongly temperature-dependent trapping into vacancies in Si, Mäkinen et al. ${ }^{10}$ estimated that the trapping coefficient at low temperatures reaches a "gigantic" value of $10^{17}$ to $10^{18} \mathrm{~s}^{-1}$.

Positron trapping in semiconductors is to some extent analogous to the capture of free carriers at defect centers. $^{20,21}$ The capture processes of electrons and holes have been extensively studied in semiconductor physics for decades. There are, however, important differences. The density of free carriers is nonzero whereas there is only one positron in the sample at a time. The singleparticle picture for a positron is thus more obvious. Secondly, band-structure effects for thermalized positrons are much less severe than for electrons and holes in several valleys with effective masses differing considerably from unity. In order that trapping by a vacancytype defect is actually seen in positron lifetime experiments a positron has to reach its ground state in the defect which is a rather localized state separated from the possible Rydberg states. This implies also that each stage in the trapping process has to be faster than the relevant positron annihilation rate $\left(\sim 10^{10} \mathrm{~s}^{-1}\right)$ in the final trapped state. In the case of free carriers, trapping at defects only involves sticking into a localized excited state and the concomitant loss of mobility. There is no need for the carriers to reach the ground state as there is for positrons. Direct mobility measurements for positrons, on the other hand, are very difficult, but useful indirect information is obtained from back-diffusion studies utilizing variable energy positron beams.

In this paper we present results from calculations for positron trapping in vacancies and negative ions in semiconductors. We rely on recent first-principles calculations ${ }^{3}$ for electron and positron states in semiconductors, and model the relevant trap potentials and wave functions so as to make explicit numerical calculations of the trapping rates feasible. First, we consider positron trapping processes in which a delocalized positron is trapped into the ground state at a vacancy. We examine two energy-loss processes in which electrons carry away the energy released by the positrons: electron-hole excitation (i) from the valence band to the conduction band and (ii) from the localized defect-associated levels to the conduction band. Secondly, we investigate a (iii) phononassisted capture in which a delocalized positron is trapped into a Rydberg state at a negative vacancy or ion. Thirdly, we study two capture processes in which a weakly localized positron makes a transition into a deeper localized state. The first of these processes is (iv) phonon assisted and concerns transitions between Rydberg states at negative vacancies and ions. The second takes place at a negative vacancy ( $v$ ) from a Rydberg state to the ground state and is mediated by the electronhole excitation process (i). The temperature dependencies of the five transition mechanisms are considered. Finally, we propose a two-state capture model which describes positron trapping into negative vacancies at low temperatures. In this model a positron is first trapped into a Rydberg state by the emission of a single phonon and thereafter the transition into the ground state occurs via the electron-hole excitation process (i) or (ii).

\section{THEORY}

Positrons implanted with a high energy of about 1 $\mathrm{MeV}$ into metals thermalize within a few picoseconds through various excitation processes. ${ }^{6,7}$ This is also true when the target medium is a semiconductor. ${ }^{22}$ At high positron energies, ionizations and electron-hole excitations dominate the energy loss. When the positron kinetic energy is lowered to approximately $0.5-1.0 \mathrm{eV}$ coupling to the phonons starts to take over. Because in semiconductors the energy gap is of similar magnitude, the cutoff of the electron-hole excitations due to the energy gap is not very important. Therefore positrons reach thermal equilibrium within a few picoseconds also in semiconductors, and the starting point for trapping into defects is a thermal equilibrium distribution for delocalized positron states. In the present calculations we use the Maxwell-Boltzmann distribution.

In this work we calculate the transition rates from delocalized positron states to the localized ones, i.e., the trapping coefficients for the different capture processes (i), (ii), and (iii) and the rates for the transitions between two localized states, (iv) and (v). We described these processes at the end of the Introduction and they are also illustrated schematically in Fig. 1. For the capture processes in the transition-limited region the trapping coefficient is given to first order by Fermi's golden-rule formula (atomic Hartree units are used) ${ }^{23}$

$$
v=4 \pi \sum_{i, f} P_{i} P_{f}\left|M_{i, f}\right|^{2} \delta\left(E_{i}-E_{f}\right),
$$

where $P_{i}$ is the probability that the initial combined positron-host state $|i\rangle$ is occupied and $P_{f}$ the probability that the final state $|f\rangle$ is allowed (in the case of electronhole excitations the final electron state has to be unoccupied). $M_{i, f}$ is the matrix element of the interaction potential taking care of the energy transfer from the positron to the host and is calculated between the initial and 


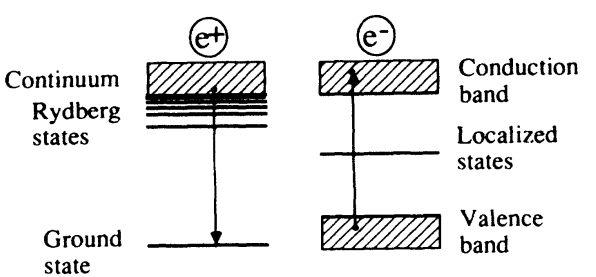

(i)

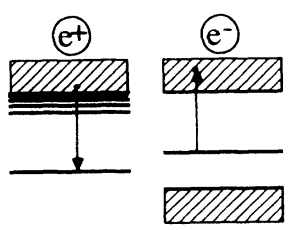

(ii)

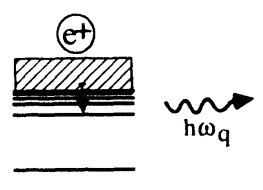

(iii)
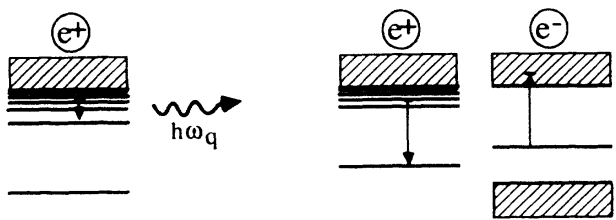

(iv)

(v)

FIG. 1. Positron capture (i), (ii), and (iii) and transition processes (iv) and ( $v$ ) with energy-loss mechanisms.

final states. The summations are extended over all possible initial and final states fulfilling the energy conservation. When the initial positron distribution is described by the Maxwell-Boltzmann form the trapping coefficient for a given temperature $T$ is calculated as the average

$$
v(T)=\frac{2}{\pi^{1 / 2}\left(k_{B} T\right)^{3 / 2}} \int_{0}^{\infty} d E v(E) e^{-E / k_{B} T} \sqrt{E},
$$

where $k_{B}$ is the Boltzmann constant and $m_{+}$the positron effective mass. The positron effective mass is assumed in all calculations of this work to be equal to the freepositron mass $\left(m_{+}=1\right.$ a.u.). The transition rates between two localized states are also given by Eq. (1) (see Sec. II e).

In a sample containing one type of positron traps, the populations of delocalized and trapped positron states are conventionally described by the kinetic equations of the so-called two-state trapping model. ${ }^{24}$ Three independent parameters are used in the equations: (i) the trapping rate $\kappa$ giving the probability per unit of time that a positron gets trapped, and the annihilation rates in (ii) the delocalized state and in (iii) the localized state. In the transition-limited regime, $\kappa$ is proportional to the concentration $c_{v}$ of the traps. The factor of proportionality is the trapping coefficient $v$. Moreover, if the binding energy of the positron to the trap is low (a shallow trap), the escape (detrapping) of the positron back to a delocalized state has to be taken into account at nonzero temperatures. When the delocalized and the trapped states are in thermal equilibrium (the principle of detailed balance), the detrapping rate $\delta$ and the trapping rate $\kappa$ at a given temperature $T$ are related as ${ }^{25}$

$$
\frac{\delta}{\kappa}=\frac{1}{c_{v}}\left(\frac{m_{+} k_{B} T}{2 \pi \hbar^{2}}\right)^{3 / 2} e^{-E_{b} / k_{B} T} .
$$

Above, $E_{b}$ is the positron binding energy in the (shallow) trap.

In order to perform numerically the various integrations implied by Eq. (1), we need to make explicit the interaction potential and to define the initial and final positron and host wave functions. We describe first the model which we use in calculating the positron wave functions and then for each capture mechanism we detail the models for the interaction potential and the host wave functions. We take the host semiconductor in our calculations to mimic $\mathrm{Si}$ and as the traps we consider the $\mathrm{Si}$ vacancy in its four charge states $+, 0,-$, and $2-$. Some of the results are also representative for negative acceptors like boron.

\section{A. Positron states}

In the case of the capture mechanisms (i), (ii), and (iii) described in Fig. 1, the initial positron wave function is delocalized whereas the final wave function is localized at the defect. The transitions (iv) and (v) occur between localized positron states at the defect. The delocalized positron states in $\mathrm{Si}$ and the localized positron ground state at the Si vacancy have been recently calculated by using a self-consistent Green-function method. ${ }^{3}$ For the present purpose it suffices to use the following simple model, the parameters of which have been chosen to match the relevant properties of positrons as calculated in Refs. 3 and 4. For the neutral vacancy, the positron states are taken to be solutions for a square-well potential (Fig. 2).

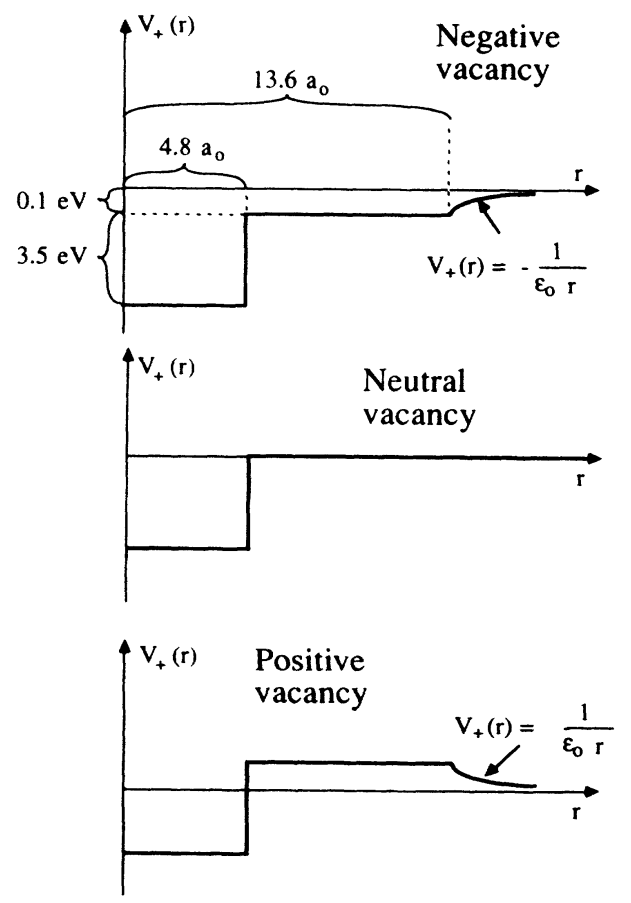

FIG. 2. Positron model potentials for singly negative, neutral, and singly positive Si vacancy. 
TABLE I. The model parameters for the positron and electron states used in the calculations. The parameters correspond to the model for neutral vacancies. The construction of potential for the charged vacancies is explained in Fig. 2. For the charged vacancies the spatial extent of the bound wave functions is practically the same as that for the neutral vacancy but the bound state energy levels are shifted by the same amount $[Q \times 0.1 \mathrm{eV}]$ as the potential near the vacancy.

\begin{tabular}{ll}
$\begin{array}{l}\text { Positron states: } \\
\text { Potential box width } \\
\text { depth }\end{array}$ & $4.8 a_{0}$ \\
$1 s$ bound state binding energy $E_{b}$ & $3.5 \mathrm{eV}$ \\
Average radius $\left\langle r_{+}\right\rangle$ & $4.94 \mathrm{eV}$ \\
Electron states: & $4.3 a_{0}$ \\
Potential box width & $6.4 a_{0}$ \\
depth & $4.2 \mathrm{eV}$ \\
$1 p$ bound state binding energy $E_{t}$ & $0.53 \mathrm{eV}$ \\
Average radius $\left\langle r_{+}\right\rangle$ & $6.0 a_{0}$ \\
\hline
\end{tabular}

The width and depth of the potential well (see Table I) are chosen so that the $s$-like ground state has a binding energy and spatial extension identical to those obtained in Refs. 3 and 4, respectively. Charged vacancies are modeled by adding a $1 / \epsilon(0) r$ tail to the square-well potential, where $\epsilon(0)$ is the static dielectric constant. The resulting potentials sensed by positrons at the negatively charged vacancy $\boldsymbol{V}^{-}$and the positively charged vacancy $V^{+}$are shown in Fig. 2. The Coulomb tail has to be cut off when approaching the vacancy from infinity in order to mimic the weak localization of the "extra" electron charge. Therefore near the vacancy we shift the potential by the amount of $Q \times(0.1 \mathrm{eV}),{ }^{26}$ where $Q$ is the charge state of the vacancy and $0.1 \mathrm{eV}$ the value of the Coulomb potential at the cutoff. This constant potential shift does not change the positron localization in the bound state and the energy eigenvalue is simply shifted by the same amount as the potential. But the potential shift and the Coulomb tail can have a large effect on the delocalized positron wave functions at thermal energies.

The delocalized positron states are scattering states in the above potentials. In the case of the state with a wave vector $\mathbf{p}$ we write

$$
u_{i, \mathbf{p}}(\mathbf{r})=\frac{4 \pi}{\sqrt{\Omega}} \sum_{l, m} i^{l} e^{i \delta_{l}}\left[Y_{l}^{m}(\hat{\mathbf{p}})\right]^{*} Y_{l}^{m}(\hat{\mathbf{r}}) R_{l, p}(r),
$$

where $\Omega$ is the normalization volume and $Y_{l}^{m}$ 's are spherical harmonics. $R_{l p}$ is the radial function solved for the square well in question. The solution of the Schrödinger equation also gives the phase shifts $\delta_{l}$. The final bound positron state wave function is denoted as

$$
u_{f}(\mathbf{r})=Y_{l^{\prime}}^{m^{\prime}}(\widehat{\mathbf{r}}) T_{l^{\prime}}(r)
$$

It is important that as an exact solution for the potential well the delocalized state shows scattering resonances as a function of the positron energy. At a resonance the positron trapping coefficient can enhance even orders of magnitude relative to the off-resonance values. ${ }^{16,17}$

In the practical calculations of this work the partial waves in Eq. (1) have been obtained by integrating numerically the Schrödinger equation from the origin outwards. The normalization of the delocalized wave functions and the determination of the phase shift have been done by fitting the integrated function to the asymptotic solution at a given cutoff radius. The asymptotic solution for a neutral vacancy is a plane wave whereas in the case of charged vacancies it is a Coulomb wave.

\section{B. Interband electron-hole excitation}

In the case of the interband electron-hole excitation the energy given by a positron in the trapping process raises an electron from the valence band to the conduction band. In a real semiconductor the valence and conduction bands have complicated structures (e.g., light- and heavy-hole bands) due to the discrete lattice structure with several ions in the unit cell. Moreover, the minimum band gap may be, as in the case of $\mathrm{Si}$, an indirect band gap. Our purpose being to obtain the qualitative features of the interband electron excitation, we neglect all these complications and describe the electrons by the following simplified model illustrated schematically in Fig. 3. The valence and conduction bands are taken as parabolic, separated by the energy gap $E_{g}$, and described by effective masses $m_{v}$ (valence band) and $m_{c}$ (conduction band). The maximum $\mathbf{k}$ vector of the valence band has the magnitude of $k_{v}=\left(3 \pi^{2} n_{v}\right)^{1 / 3}$ determined by the valence electron density $n_{v}$, in Si. The initial and final electron states in this process are thus considered as plane waves. In the transitions the electron $\mathbf{k}$ vectors of the initial and final states may differ from each other.

Within the above model the transition rate (1) can be calculated as

$v(\mathbf{p})=4 \pi \sum_{\mathbf{k}} \sum_{\mathbf{q}}|M(\mathbf{p}, \mathbf{q})|^{2} \Theta\left(k_{v}-k\right) \delta\left(E_{i}-E_{f}\right)$,

where the $\mathbf{k}$ summation runs over the initial electron $\mathbf{k}$ values in the valence band and $q$ is the momentum transfer. $\Theta$ is the step function. A straightforward cal-

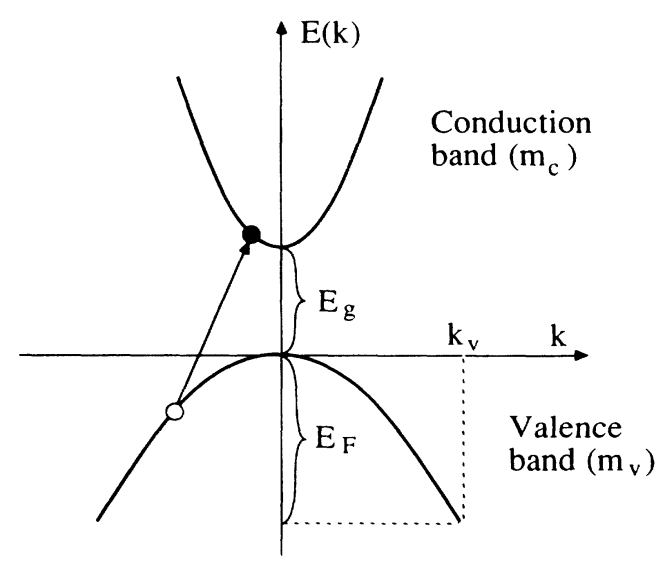

FIG. 3. Interband electron-hole excitation. 
culation gives

$$
\begin{aligned}
v(\mathbf{p})=4 \pi \frac{\Omega}{(2 \pi)^{3}} \int d q q^{2} \int d \Omega_{\hat{\mathbf{q}}}|\boldsymbol{M}(\mathbf{p}, \mathbf{q})|^{2} \frac{\Omega}{(2 \pi)^{3}} \\
\times \int_{0}^{k_{v}} d k 2 \pi k^{2} \frac{m_{c}}{k q} \Theta(z-|Z|),
\end{aligned}
$$

where

$$
z=\frac{k q}{m_{c}}
$$

and

$$
Z=\frac{p^{2}}{2 m_{+}}-\frac{k^{2}}{2 m_{v}}+E_{b}-E_{g}-\frac{k^{2}}{2 m_{c}}-\frac{q^{2}}{2 m_{c}} .
$$

In order to get the trapping coefficient (i.e., the trapping rate per unit defect concentration in the transitionlimited regime) the normalization volume $\Omega$ has to be chosen as the volume per atom in the lattice.

The matrix element in Eq. (7) reads

$$
M_{i, f}=\frac{1}{\Omega} V(\mathbf{q}) \int d \mathbf{r} u_{i, \mathbf{p}}^{*}(\mathbf{r}) u_{f}(\mathbf{r}) e^{i \mathbf{q} \cdot \mathbf{r}},
$$

where $V(q)$ is the Fourier transform of the screened Coulomb potential describing the positron-electron interaction. Inserting the expansion $\left(j_{l}\right.$ is the spherical Bessel function)

$$
e^{i \mathbf{q} \cdot \mathbf{r}}=4 \pi \sum_{l^{\prime \prime}, m^{\prime \prime}} i^{l^{\prime \prime}} Y_{l^{\prime \prime}}^{m^{\prime \prime} *}(\hat{\mathbf{q}}) Y_{l^{\prime \prime}}^{m^{\prime \prime}}(\hat{\mathbf{r}}) j_{l^{\prime \prime}}(q r)
$$

and the initial and final positron wave functions, i.e., Eqs. (4) and (5), in Eq. (10) and integrating over the angles of $\mathbf{r}$ and $\mathbf{q}$ we obtain

$$
\int d \Omega_{\hat{\mathbf{q}}}\left|M_{l^{\prime}}(\mathbf{p}, \mathbf{q})\right|^{2}=\frac{|4 \pi V(q)|^{2}}{\Omega^{3}} A_{l^{\prime}},
$$

where

$$
\begin{gathered}
A_{l^{\prime}}=\sum_{l}\left[(2 l+1)\left[\int_{0}^{\infty} d r r^{2} R_{l p}^{*}(r) T_{0}(r) j_{l}(q r)\right]^{2}\right] \\
\text { when } l^{\prime}=0 ; \\
A_{l^{\prime}}=3 \sum_{l}\left[l\left[\int_{0}^{\infty} d r r^{2} R_{l p}^{*}(r) T_{1}(r) j_{l-1}(q r)\right]^{2}\right. \\
+(l+1)\left[\int_{0}^{\infty} d r r^{2} R_{l p}^{*}(r) T_{1}(r)\right. \\
\left.\left.\times j_{l+1}(q r)\right]^{2}\right], \text { when } l^{\prime}=1 .
\end{gathered}
$$

Above, the summation over the final positron state $m^{\prime}$ quantum numbers has been performed.

For the electron-positron Coulomb interaction we use the statically screened form

$$
V(\mathbf{q})=\frac{1}{\epsilon(\mathbf{q})} \frac{4 \pi}{q^{2}},
$$

with the wave-vector-dependent dielectric function $\epsilon(\mathbf{q})$ suggested by Penn ${ }^{27}$

$$
\epsilon(\mathbf{q})=1+\left(\omega_{p} / \bar{E}_{g}\right)^{2} F\left[1+\left(E_{F} / E_{g}\right)\left(q / k_{F}\right) F^{1 / 2}\right]^{-2},
$$

where $F=1-\frac{1}{4}\left(\bar{E}_{g} / E_{F}\right)$. The Fermi energy $E_{F}$ and Fermi momentum $k_{F}$ as well as the plasma frequency $\omega_{p}$ are calculated in the model from the average valence electron density. $\bar{E}_{g}$ is an "average" band gap determined so that $\epsilon(0)$ calculated from Eq. (16) is equal to the experimental static dielectric constant.

\section{Electron excitation from localized states at the vacancy}

Vacancy-type defects in semiconductors induce bound electron states localized around the vacancy and with energies located in the band gap. We have studied positron trapping processes in which these bound electrons are excited into the conduction band. We consider the case of the Si vacancy and in order to describe the localized electron states we make the same simplified model as for the positron states (see Sec. II A): The neutral vacancy $V^{0}$ is a square well and the charged vacancies $V^{+}$and $V^{-}$are square wells with an additional $1 / \epsilon(0) r$ tail. The parameters of the well used for the electrons are given in Table I. They are chosen so that the first $p$ state of the square well reproduces the binding energy and the spatial extension calculated in Ref. 3 for the bound $T_{2}$ state induced by the vacancy in the $T_{d}$ symmetry of the diamond lattice. The $T_{2}$ state vanishes at the center of the vacancy and its wave function has maxima in the region of the dangling bonds of the four Si atoms surrounding the vacancy. In the case of charged vacancies the bottom of the potential well is shifted by the amount of $-Q \times(0.1 \mathrm{eV})$ analogously to the changes in the positron potential (see Sec. II A). This shift is directly transferred to the binding energies of the localized electrons.

The trapping coefficient is then written as

$$
v(\mathbf{p})=\frac{N_{d} m_{c} \Omega k}{6 \pi^{2}} \int d \Omega_{\hat{\mathbf{k}}} \sum_{m}\left|{ }_{1}^{m} M\right|^{2}
$$

where $N_{d}$ is the number of deep level electrons and $k$ is the electron wave vector in the final state. Due to energy conservation it has the magnitude

$$
k=\left[2 m_{c}\left(\frac{p^{2}}{2 m+}-E_{i}+E_{b}\right)\right]^{1 / 2},
$$

where $E_{i}$ is the deep level energy relative to the bottom of the conduction band. The integration in Eq. (17) is performed over possible orientations of the electron $\mathbf{k}$ vector in the final state and the summation runs over the different $m$ quantum numbers of the initial $p$ state (i.e., $m=-1,0,+1)$. The matrix element ${ }_{1}^{m} M$ in Eq. (17) reads

$$
{ }_{1}^{m} M=\frac{1}{(2 \pi)^{3}} \int d \mathbf{q}^{\prime} V\left(\mathbf{q}^{\prime}\right) I_{+}\left(\mathbf{q}^{\prime}\right){ }_{1}^{m} I_{-}\left(\mathbf{q}^{\prime}\right) .
$$

Above, $I_{+}$and ${ }_{1}^{m} I_{-}$denote the integrals

$$
I_{+}\left(\mathbf{q}^{\prime}\right)=\int d \mathbf{r} u_{i, \mathbf{p}}^{*}(\mathbf{r}) e^{i \mathbf{q}^{\prime} \cdot \mathbf{r}} u_{f}(\mathbf{r})
$$


and

$$
{ }_{1}^{m} I_{-}\left(\mathbf{q}^{\prime}\right)=\int d \mathbf{r}_{1}^{m} v_{i}^{*}(\mathbf{r}) e^{i \mathbf{q}^{\prime} \cdot \mathbf{r}} v_{f, \mathbf{k}}(\mathbf{r}) .
$$

In Eq. (21) the initial $\left[u_{i, \mathbf{p}}(\mathbf{r})\right]$ and final $\left[u_{f}(\mathbf{r})\right]$ positron states are as above in the case of the interband transitions, i.e., they are obtained from the potential well given in Fig. 2. The initial electron wave function ${ }_{1}^{m} v_{i}(\mathbf{r})$ is a bound $p$ state similar to Eq. (5) and the final electron wave function $v_{f, \mathbf{k}}(\mathbf{r})$ is a scattering state similar to Eq. (4). The initial states are calculated for the vacancy with a charge $Q$, the final states are calculated for the vacancy with the charge $Q+1$ since in the final state the vacancy has lost an electron. The angular integrals over $\Omega_{\mathbf{k}}, \Omega_{\hat{\mathbf{q}}^{\prime}}$, and $\Omega_{\widehat{\mathrm{r}}}$ can be performed analytically, but the radial $q$ and $r$ integrals have to be calculated numerically.

\section{Positron trapping into Rydberg states via phonon emission}

The long-range attractive Coulomb tail of the negative vacancies supports weakly bound Rydberg states for positrons. The positron binding energies for these states are typically much smaller than the band gap so that the electron excitation processes discussed above are unavailable for mediating the trapping. However, as the binding energies are smaller than the Debye energy, a process in which one phonon is emitted is possible. The trapping coefficient for a phonon process has been derived previously for longitudinal-acoustic phonons using the deformation potential and Thomas-Fermi-screening models. ${ }^{28}$ For a general case, the equation reads as

$$
v_{l^{\prime}}(\mathrm{p})=\frac{4 E_{d}^{2}[V(q)]^{2} q^{3} k_{F}^{2}}{\pi^{4}(2 \pi)^{6} s^{2} p \Omega}[n(q, T)+1] A_{l^{\prime}},
$$

where $V(q)$ is now the Penn-screened Coulomb potential given in Eq. (15). The wave vector $q$ has the value

$$
q=\frac{p^{2} / 2 m+E_{b}}{s},
$$

and the factor $A_{l^{\prime}}$ is defined in Eq. (13) or (14) with $T_{0}$ and $T_{1}$ referring now to Rydberg states. $E_{d}$ is the positron deformation potential constant, $s$ the sound velocity, $\rho$ the mass density, and $n(q, T)$ is the Bose-Einstein distribution function for longitudinal-acoustic phonons.

\section{E. Transitions between bound positron states}

Fermi's golden rule [Eq. (1)] can naturally be used to calculate also the transition rates between two localized positron states. In the case of the negative vacancy $V^{-}$ we consider the transitions between different Rydberg states or between a Rydberg state and the ground state. In the practical calculations it suffices to use in Eq. (12) or (13) for the initial state (with angular momentum $l$ ) the bound state radial wave function instead of $R_{l p}(r)$, omit the summation over $l$, and multiply the expression by $\Omega /[4 \pi(2 l+1)]$.
TABLE II. Host parameters mimicking Si used in the calculations.
Volume per atom $\Omega$

Number of valence electrons per atom

Electron energy band gap $E_{g}$

Static dielectric constant $\epsilon(0)$

Velocity of the longitudinal

acoustic waves $s$

Mass density $\rho$

Positron deformation potential $E_{d}$
$136 a_{0}^{3}$

4

$1.12 \mathrm{eV}$

11.7

$9130 \mathrm{~m} / \mathrm{s}$

$2.33 \mathrm{~g} / \mathrm{cm}^{3}$

$6.2 \mathrm{eV}$

\section{CAPTURE MECHANISMS INVOLVING ELECTRON EXCITATION}

\section{A. Interband electron-hole excitation}

We begin the presentation of our results with the case of the neutral vacancy and then discuss the results for the negative and positive vacancies. In the calculations for the neutral vacancy, the initial and final positron states correspond to the potential box with parameters given in Table I. The positron binding energy in the final localized state is $0.94 \mathrm{eV}$. This implies that in order to satisfy the energy conservation law, the interband transition must take place in a semiconductor with a band gap smaller than $0.94 \mathrm{eV}$. The parameters of the generic semiconductor we consider for this section are given in Table II. They are taken from $\mathrm{Si}$ with the exception of the electron band gap and the effective band masses which are chosen as variable parameters.

Firstly, we present in Fig. 4 the dependence of the trapping coefficient on the width of the band gap. The calculations have been made for the temperature of 100 $K$ and for the effective valence and conduction electron masses equal to the free-electron mass. The trapping coefficient decreases linearly from $10^{15} \mathrm{~s}^{-1}$ as the energy gap increases and vanishes at the value of $0.94 \mathrm{eV}$ when

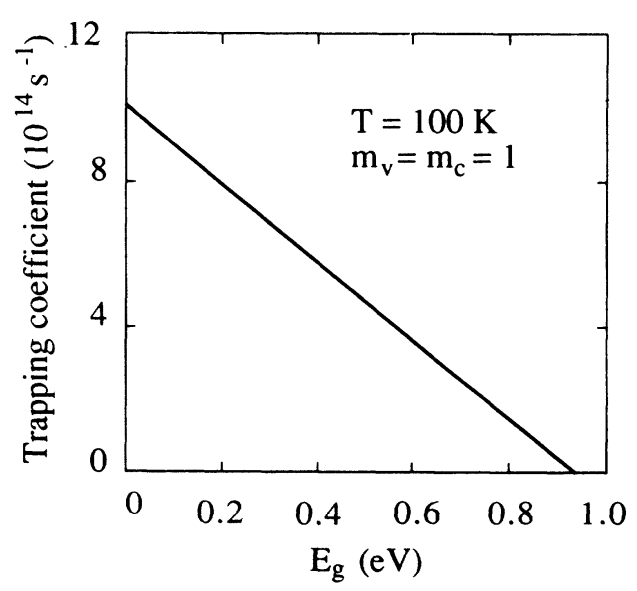

FIG. 4. Positron trapping coefficient vs the width of the band gap in the interband electron-hole excitation process [(i) in Fig. 1]. The positron states are calculated using the model potential for a neutral vacancy (Fig. 2). The results correspond to the temperature of $100 \mathrm{~K}$ and the electron mass for the valence and conduction band is equal to the free-electron mass. 


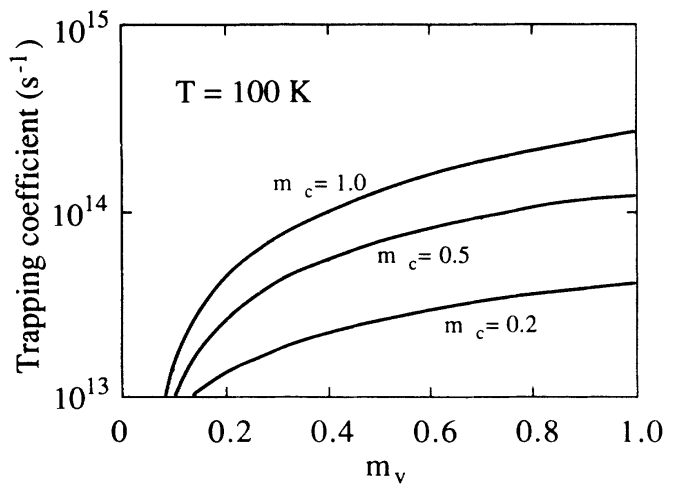

FIG. 5. Dependence of the positron trapping coefficient on the effective electron masses in the interband electron-hole excitation process [(i) in Fig. 1]. The trapping coefficient is shown as a function of the effective valence-band mass $m_{v}$ for three different conduction-band masses $m_{c}$. The positron states are calculated using the model potential for a neutral vacancy (Fig. 2). The results correspond to the temperature of $100 \mathrm{~K}$ and the band gap is $0.7 \mathrm{eV}$.

the gap equals the positron binding energy. The values of the order of $10^{15} \mathrm{~s}^{-1}$ at the zero gap are similar to those found in metals. ${ }^{6,7}$

Secondly, we show in Fig. 5 the effects of varying the effective electron masses with the band gap fixed arbitrarily at $E_{g}=0.7 \mathrm{eV}$, a value which is slightly smaller than the positron binding energy in the neutral vacancy. The trapping coefficient decreases from $4 \times 10^{14} \mathrm{~s}^{-1}$ to $10^{13} \mathrm{~s}^{-1}$ when either of the band masses decreases from 1 to 0.1 a.u. This behavior reflects the fact that the phase space allowed for the transition shrinks when either of the band masses decreases. Moreover, the decrease of the trapping coefficient is symmetric with respect to changes of the valence- and conduction-band masses. The main conclusion from Fig. 5 is that the use of realistic band masses $[(0.2-0.4) \times($ free-electron mass $)]$ decreases the

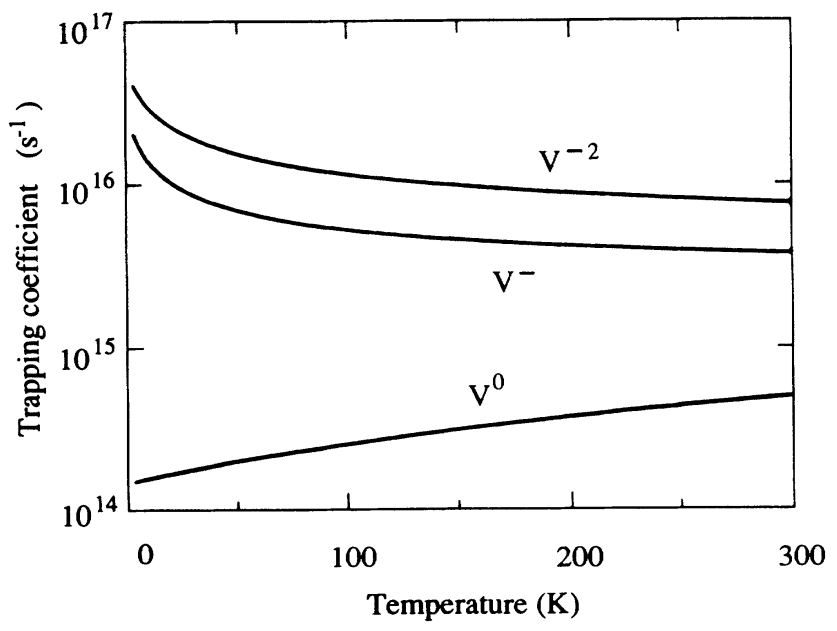

FIG. 6. Temperature dependence of the positron trapping coefficient for neutral $\left(V^{0}\right)$, singly negative $\left(V^{-}\right)$, and doubly negative $\left(V^{2-}\right)$ vacancies. The interband electron-hole excitation process [(i) in Fig. 1] with positron states from the model potentials of Fig. 2 is used. The electron mass for the valence and conduction band is equal to the free-electron mass. trapping coefficient: the value obtained by using freeelectron masses has to be divided typically by a factor of 10.

Figure 6 shows the temperature dependence of the trapping coefficient for the neutral vacancy $V^{0}$. In this calculation the electron band gap is fixed to be $0.7 \mathrm{eV}$ and the electron band masses are equal to the free-electron mass. According to Fig. 6 the trapping coefficient for a neutral vacancy shows an increasing trend as temperature rises from 0 to $300 \mathrm{~K}$ : it increases by a factor of 3 from 1.5 to $5 \times 10^{14} \mathrm{~s}^{-1}$. This increase is due to a $p$-type scattering resonance for delocalized positron states at energies above the typical thermal energies. If the positron kinetic energy is near the resonance the initial positron state is strongly enhanced at the vacancy, which increases the overlap of the initial and final states and thus also the positron trapping coefficient. When temperature rises, the kinetic energy of the positron increases and the contribution from the resonance region to the thermal average integral [Eq. (2)] becomes more important causing the trend seen in Fig. 6.

For the negative vacancy $\mathrm{V}^{-}$, the initial and final positron states are calculated using the potential with the attractive Coulomb tail (Fig. 2) and the initial scattering states are then Coulomb waves. At $300 \mathrm{~K}$, the trapping coefficient is about $4 \times 10^{15} \mathrm{~s}^{-1}$, which is about one order of magnitude larger than the coefficient for the neutral vacancy. The difference increases towards lower temperatures as shown in Fig. 6. The trapping coefficient for the negative vacancy diverges at low temperatures being proportional to $T^{-1 / 2}$ and at $5 \mathrm{~K}$ it reaches the value of $5 \times 10^{16} \mathrm{~s}^{-1}$. This divergence is a direct consequence of the normalization of the initial positron wave function. The square of the amplitude of the Coulomb wave at the origin (i.e., in the center of the vacancy) is at maximum and it behaves as ${ }^{29}$

$$
\left|u_{i, \mathrm{p}}(0)\right|^{2} \propto \frac{\alpha}{e^{2 \pi \alpha}-1},
$$

where

$$
\alpha=\frac{m+Q}{\epsilon(0) p} .
$$

For a negative charge state $Q$, the square of the matrix element (10) and the ensuing positron trapping coefficient are inversely proportional to the square root of the positron energy. In Eq. (2), the integral over energy becomes then proportional to temperature and consequently, due to the prefactor proportional to $T^{-3 / 2}$, the resulting trapping coefficient is proportional to $T^{-1 / 2}$. For $\alpha$ values close to zero the amplitude in Eq. (24) approaches a constant value and the wave function becomes a plane wave. Thus neutral vacancies do not produce a trapping coefficient diverging at low temperatures. Moreover, it is seen from Eq. (24) that for a positively charged vacancy the initial positron wave function at the vacancy and therefore also the trapping coefficient should be vanishingly small. The further discussion concerning positive vacancies is postponed to the next subsection dealing with the electron excitation from the localized levels.

For the doubly negative vacancy $V^{2-}$, the trapping 
coefficient diverges also as $T^{-1 / 2}$. According to Eq. (24), the trapping coefficient is directly proportional to $Q$ so that the ratio of the trapping coefficients for $V^{2-}$ and $V^{-}$ should be a factor of 2 . This is obeyed very well by the actual calculated results shown in Fig. 6.

One should bear in mind that the trapping coefficients for the interband electron excitation processes shown in Fig. 6 are upper-limit estimations. In the case of the wide-gap semiconductors the gap may be of the same order as or even larger than the positron binding energy in the defect. The increase of the band gap diminishes strongly the trapping coefficient (Fig. 4). The trapping coefficient is also expected to be small in narrow-gap semiconductors in which the electron band masses are much smaller than unity (Fig. 5). Only in those narrowgap semiconductors in which electron band masses are close to unity, can the trapping coefficient become roughly as large (neutral vacancies) or larger (negative vacancies) than that calculated for vacancies in metals. ${ }^{17}$ The two major differences between the present calculations and similar calculations describing metallic systems follow: (i) The phase space for allowed transitions is determined by valence and conduction bands in semiconductors, which are a parabola opening downwards and upwards, respectively, whereas a metal can be mimicked by a parabolic free-electron band and the excitations take place over the Fermi surface. This difference in the topology of the phase space favors trapping in metals as compared to semiconductors. (ii) The screening of the electron-positron interaction is less efficient in semiconductors than in metals: the Penn model [Eq. (16)] is used in semiconductors instead of the Thomas-Fermi screening in metals. The less efficient screening tends to increase the trapping coefficient. The trapping coeffcient is smaller approximately by a factor of 10 if the ThomasFermi screening is used instead of the Penn model.

\section{B. Excitation of localized electrons: Ionization of the vacancy}

The excitation of electrons from localized defect levels is expected to be a more efficient way to dissipate the positron energy in the trapping process than the interband excitations because the initial electron wave functions involved are localized near the vacancy. On the other hand, there are now only a few discrete levels available whereas in the case of interband excitations there is a continuum of delocalized initial electron states. The results in this subsection will show that the localization compensates the limited number of available states, and therefore there exists an efficient trapping mechanism also in wide-gap semiconductors. The present calculations are based on the localized and delocalized electron and positron states constructed by using the potential wells described in Table I for the neutral vacancy and represented in Fig. 2 for the positive and negative vacancy. The effective electron mass in the conduction band is in the calculations equal to the free-electron mass.

The temperature dependence of the trapping coefficient for a neutral vacancy is plotted in Fig. 7. The trapping coefficient for a neutral vacancy is practically independent of temperature. The $s$ contribution dominates over

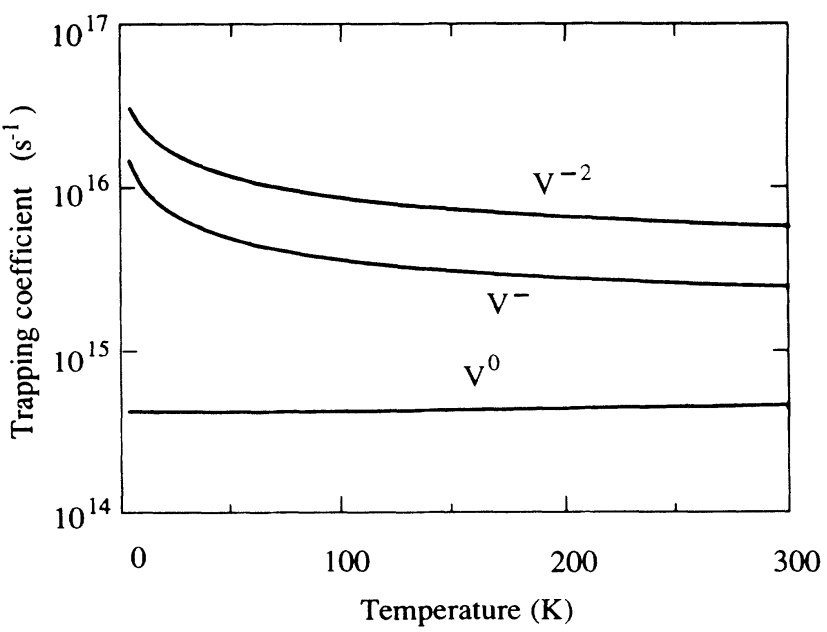

FIG. 7. Temperature dependence of the positron trapping coefficient for neutral $\left(V^{0}\right)$, singly negative $\left(V^{-}\right)$, and doubly negative $\left(V^{2-}\right)$ vacancies. The process of electron excitation from the localized states [(ii) in Fig. 1] is used. Positron and electron states are constructed according to the model potentials of Fig. 2 and parameters in Table I.

the higher $l$ components in the sum over the positron angular momentum quantum numbers in the equation for the matrix element. Thus, the $p$ resonance for delocalized positron states has little effect on the trapping coefficient in this case in contrast with the case of the interband excitations. The magnitude of the trapping coefficient is about $4 \times 10^{14} \mathrm{~s}^{-1}$, which is of similar order as that for the interband excitation at $300 \mathrm{~K}$ in Fig. 6 .

The temperature dependences of the trapping coefficient for the singly and doubly negative vacancies are also shown in Fig. 7. They are similar to those observed for the interband excitation capture: They show the $T^{-1 / 2}$ divergences due to the character of the Coulomb waves. The trapping coefficients at $300 \mathrm{~K}$ are now about $2.5 \times 10^{15} \mathrm{~s}^{-1}$ and $6 \times 10^{15} \mathrm{~s}^{-1}$ for $V^{-}$and $V^{2-}$, respectively. These values are very similar to the corresponding values shown for the interband excitation process in Fig. 6. Because the trapping coefficient is now proportional to the number of bound localized electrons (four for $V^{2-}$ and three for $V^{-}$), a simple estimation based on Eq. (24) would give the ratio of $\sim 2.7$ between the trapping coefficients for the doubly negative and singly negative vacancies. The actual calculation gives a slightly smaller value.

The temperature dependence of the trapping coefficient for a positively charged vacancy is plotted in Fig. 8 . The coefficient falls to zero as temperature decreases. This is because according to Eq. (24) the positron wave function near the vacancy vanishes exponentially as the positron kinetic energy decreases. According to Fig. 8 trapping into positively charged vacancies before annihilation could be possible at temperatures higher than $\sim 200 \mathrm{~K}$. However, only for very high vacancy concentrations of the order of $c \approx 10^{-2}$ atom ${ }^{-1}$ positron trapping rate $\kappa=c v$ at the highest tempeatures shown in Fig. 8 is larger 


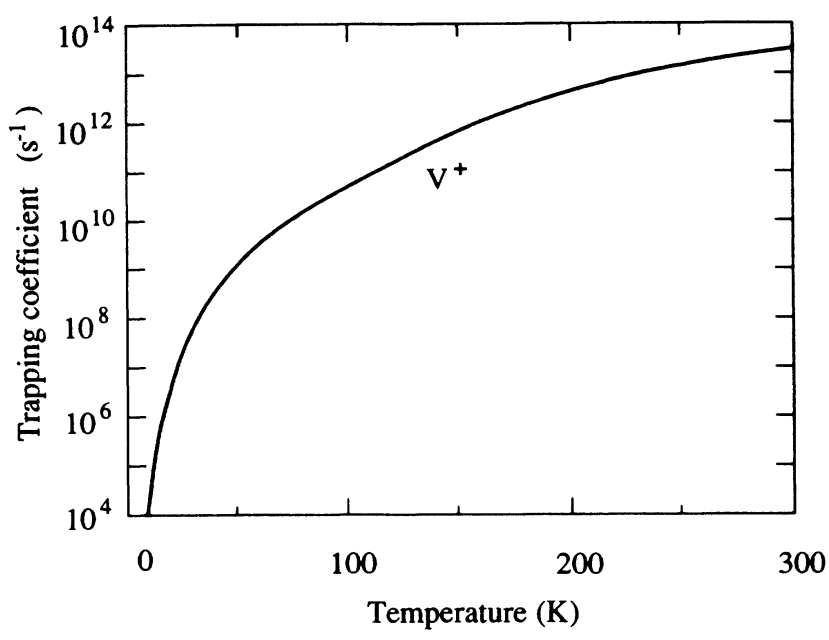

FIG. 8. Temperature dependence of the positron trapping coefficient for a positive $\left(V^{+}\right)$vacancy. The process of electron excitation from the localized states [(ii) in Fig. 1] is used. Positron and electron states are constructed according to the model potentials of Fig. 2 and parameters in Table I.

than the typical positron annihilation rates in semiconductors which are of the order of $\sim 5 \times 10^{9} \mathrm{~s}^{-1}$. However, the trapping coefficient into positively charged vacancies is much smaller than the rate into neutral or negatively charged vacancies. Moreover the trapping coefficient is very sensitive to the height and width of the potential barrier surrounding the positive vacancy. For example, if the barrier height is raised from 0.1 to $0.2 \mathrm{eV}$ the trapping coefficient sinks over 4 orders in magnitude. This explains why positive vacancies have not been seen in positron lifetime experiments although the existence of a bound positron state at them is predicted theoretically. ${ }^{3}$ The positron simply does not have enough time to tunnel through the repulsive Coulomb barrier.

In the case of the neutral vacancy, we have studied the effects of varying the potential well parameters and the ensuing binding energy for the positron states. The temperature dependence of the trapping coefficients is shown in Fig. 9 for four different potential wells. The width of the well is varied while the depth is kept as a constant equal to $3.5 \mathrm{eV}$. The positron binding energy increases rather linearly from 0.51 to $1.36 \mathrm{eV}$ when the well width increases from $4.3 a_{0}$ to $5.8 a_{0}$. For the three smallest well widths, i.e., $4.3 a_{0}, 4.8 a_{0}$, and $5.8 a_{0}$ the results are very similar: the magnitude of the trapping coefficient is nearly the same in every case and it increases only slightly as a function of temperature. But when the well width is increased to $5.8 a_{0}$, the behavior changes dramatically. A $p$ resonance for the delocalized positron states is near the typical thermal energies and as a consequence the trapping coefficient rises very strongly by a factor larger than 10 when temperature increases from 0 to $300 \mathrm{~K}$.

In the case of the neutral vacancy we have also studied the effects of varying the electron states. The variations which are allowed are small because the potential well has to satisfy two requirements: (i) a bound $p$ state must

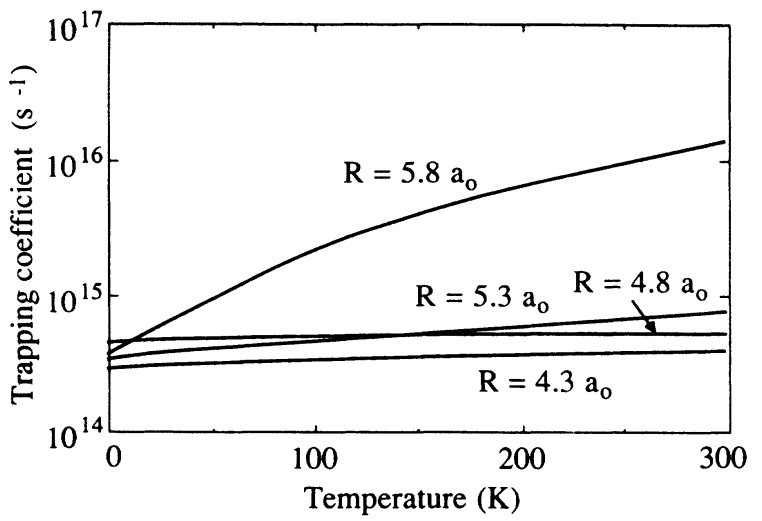

FIG. 9. Temperature dependence of the positron trapping coefficient for a neutral $\left(V^{0}\right)$ vacancy in the process of electron excitation from the localized states [(ii) in Fig. 1]. Four different potential well widths $(R)$ are used to construct the positron states. Otherwise the positron and electron states are constructed according to the model potentials of Fig. 2 and parameters in Table I.

exist and (ii) the electron binding energy to the localized level has to be smaller than the positron binding energy in the vacancy. There is no change in the temperature dependence of the trapping coefficient for the small variations we have considered. But at a given temperature, the trapping coefficient shows a strong dependence on the electron binding energy $E_{i}$. This is illustrated in Fig. 10. When the well width increases, the electron binding energy $E_{i}$ increases and the trapping coefficient decreases linearly as a function of $E_{i}$. This behavior reflects the decrease of the phase space for allowed transitions. As the electron binding energy becomes higher than the positron binding energy, the positron trapping coefficient vanishes. This behavior as a function of $E_{i}$ is similar to that observed as a function of $E_{g}$ in Fig. 4 for the case of the interband electron excitations.

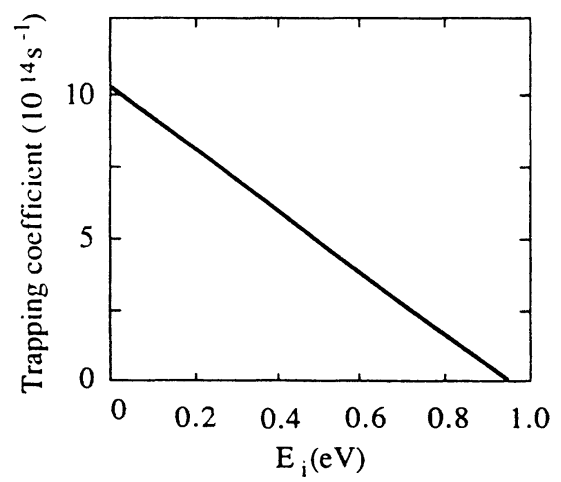

FIG. 10. Positron trapping coefficient vs the binding energy $E_{i}$ of the localized electrons in a neutral $\left(V^{0}\right)$ vacancy for the process of electron excitation from the localized states [(ii) in Fig. 1]. The binding energy is varied by changing the potential well width in constructing the electron states. Otherwise the positron and electron states are constructed according to the model potentials of Fig. 2 and parameters in Table I. 
To conclude this section: for semiconductors electron excitation from a localized state to the conduction band provides an efficient channel for positron trapping. At $300 \mathrm{~K}$, the trapping coefficient for the neutral vacancy is about $4 \times 10^{14} \mathrm{~s}^{-1}$ and the coefficient increases by a factor $\sim 4$ when its charge changes from neutral to singly negative and by another factor of 2-3 when the charge changes from singly negative to doubly negative. These ratios are in good agreement with the recent experimental estimations ${ }^{11}$ of the positron trapping coefficient for $\mathrm{Si}$ divacancies at different charge states. However, the experimental temperature dependencies are much stronger than those seen in Fig. 7. This suggests that other types of trapping mechanisms, in which detrapping is possible, should be important. But the ratio between between the trapping coefficient for the singly negative and the doubly negative defects is a property of the initial positron states in the Coulomb potential, and therefore it is expected to be valid also for other capture mechanisms.

\section{CAPTURE MECHANISMS INVOLVING PHONON EXCITATION}

\section{A. Capture from a delocalized state to a Rydberg state}

The attractive Coulomb tail in the potential for negative vacancies supports positron Rydberg states characterized by a low binding energy and a large spatial extent. The small positron binding energy makes electron-hole excitations impossible. However, because the energy release in the trapping process is usually smaller than the Debye energy, phonon emission is possible. The large spatial extent of the Rydberg state is expected to lead to large values for the matrix element between the initial scattering state and the final Rydberg state. As shown below, it is effectively the case.

In this section we consider singly negative vacancies in Si described according to Fig. 2 and Table I. The calculated trapping coefficients for $s$-type and $p$-type Rydberg states are shown in Fig. 11 as a function of the quantum number $n$. The results correspond to the temperature of $10 \mathrm{~K}$. Figure 11 shows that for a given $n$ the trapping coefficient increases when the $l$ quantum number of the final Rydberg states increases. This is mainly because of the increase of the degeneracy and the delocalization of the Rydberg wave function. The increase is less marked for larger $n$ : the factor of increase is about 100 for $n$ equal to 4 but about 10 for $n$ equal to 7 . For a given $l$, Fig. 11 shows also that the trapping coefficient increases rapidly when $n$ increases. For the $p$-type Rydberg state, the trapping coefficient increases by a factor of $\sim 100$ from $7 \times 10^{14}$ to $4 \times 10^{16} \mathrm{~s}^{-1}$ when $n$ increases from 3 to 6 . This behavior can be understood as follows. The matrix element for the positron trapping coefficient depends on the integral of the product of a Rydberg state wave function, a Coulomb wave, and a spherical Bessel function [Eqs. (13) and (14)]. Figure 12 shows typical positron wave functions for some Rydberg states and a Coulomb wave for the low kinetic energy of $25 \mathrm{meV}$. It can be seen that the functions oscillate in the phase near the origin. They are, however, orthogonal because they are driven

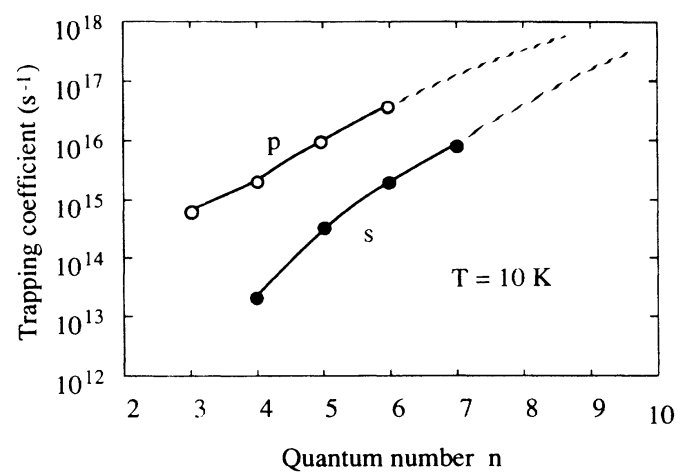

FIG. 11. Positron trapping coefficient for the $s$ - and $p$-type Rydberg states as a function of the quantum number $n$. The energy is transferred to a phonon [(iii) in Fig. 1]. The positron states are calculated using the model potential for a singly negative vacancy (Fig. 2). The results correspond to the temperature of $10 \mathrm{~K}$.

out of phase at large distances. When the quantum number $n$ increases the oscillations stay in phase to larger and larger distances. The main contribution to the total integral in the matrix element comes from the region near the origin where the Rydberg state wave functions and the Coulomb wave are in phase and the Bessel function does not yet oscillate. For large $n$ values this region becomes larger, which makes the matrix element and the positron trapping coefficient increase.

The quantum number $n$ of the Rydberg state into which positrons can be trapped has from the experimental point of view a maximum value. In a sample containing a finite vacancy concentration, this maximum value corresponds to the quantum number $n$ for which the Rydberg states belonging to neighboring vacancies start

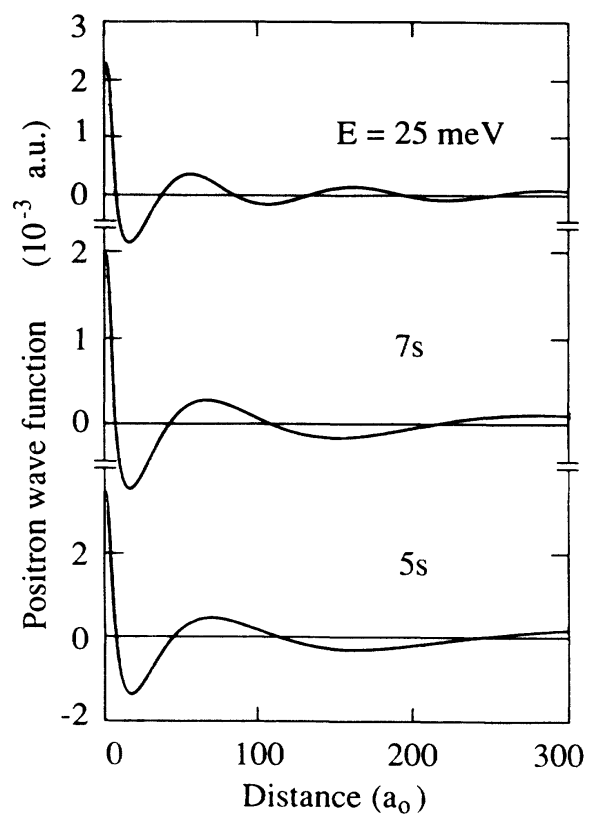

FIG. 12. Positron wave function for the $5 s$ and $7 s$ Rydberg state and a Coulomb scattering state at the energy of $25 \mathrm{meV}$. The states are calculated for the singly negative vacancy (Fig. 2). 
to overlap and form a "Rydberg band." For example, for a typical vacancy concentration of $10^{15} \mathrm{~cm}^{-3}\left(\approx 2 \times 10^{-8}\right.$ atom ${ }^{-1}$ ) the volume per vacancy is equal to the volume of the sphere with the average radius of the Rydberg state with $n \approx 10$. The quantum number $n$ can also be limited because of the screening due to the charge carriers in highly doped semiconductors. Extrapolation of the curves in Fig. 11 to an $n$ value as large as 10 would yield such "gigantic" values of the trapping coefficient as $10^{18}$ $\mathrm{s}^{-1}$.

The temperature dependence of the trapping coefficient for a Rydberg state is shown in Fig. 13. The final state is the $7 s$ state. The trapping coefficient decreases strongly when temperature increases. The decrease is caused by the properties of the Coulomb waves as discussed in the context of the interband transitions above. The other reason for the decrease of the trapping coefficient is that the integrals in Eq. (13) or (14) depend strongly on the $q$ value [Eq. (23)] appearing in the spherical Bessel function. The value of $q$ (Eq. 23) is proportional to the sum of the initial kinetic energy and final binding energy in the Rydberg state. For the Rydberg states the binding energies $E_{b}$ are small so that the contribution from the initial state kinetic energy dominates as temperature increases. When $q$ increases the Bessel function oscillates more rapidly which decreases the values of the integrals. Note also that this effect is strong because the final state is rather delocalized: if the final state were well localized at the vacancy, the Bessel function would not start to oscillate within this region until $q$ has rather a large value.

In the case of a negative vacancy, the trapping coefficient for an excited Rydberg state (Fig. 13) can be compared to coefficients obtained for trapping into the ground state (Figs. 6 and 7). At room temperature the trapping coefficients are of the same order, i.e., $10^{15}$ to $10^{16} \mathrm{~s}^{-1}$. However, detrapping occurs from the Rydberg state because of the low positron binding energy. Consequently, the probability that a positron occupies a Rydberg state is small compared to the probability that the

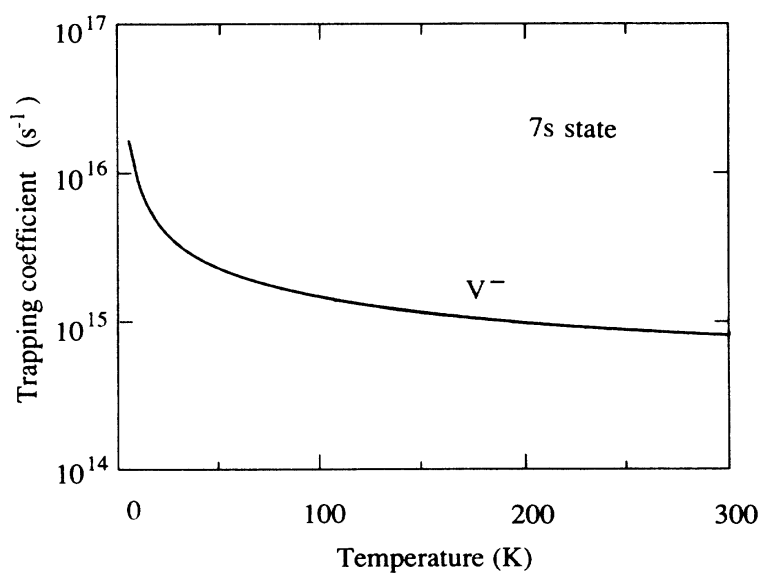

FIG. 13. Temperature dependence of the positron trapping coefficient for the 7s Rydberg state in the phonon emission process [(iii) in Fig. 1]. The positron states are calculated using the model potential for a singly negative vacancy (Fig. 2). positron occupies the ground state. Annihilation occurs in the ground state and the positron trap can be identified as a vacancy. At low temperatures, the trapping coefficients for Rydberg states are of the same order (for $n \leq 7$ ) or larger by an order of magnitude (for $n>7$ ) than for the ground state. Because at low temperatures the detrapping rate is low [see Eq. (3)] annihilation from a Rydberg state becomes highly probable. However, positron trapping at vacancies is still experimentally observed at 4 K. ${ }^{10}$ This means that a positron does not annihilate in a Rydberg state but it makes a transition to the ground state once it has been trapped into a Rydberg state. Two ways are in principle possible. Either (i) positrons reach the ground state through successive transitions between Rydberg states in a mechanism analogous to the Lax cascade $^{30-32}$ suggested for the carrier capture at charged centers or (ii) positrons are directly trapped into the ground state. The transition rates for these processes are calculated in this work and discussed in the next subsections.

The energy eigenvalues and the wave functions of the Rydberg states calculated for the charged vacancy become independent of the shape of the potential near the origin when the quantum number $n$ increases. Therefore also the trapping coefficients for the Rydberg states and the transition rates between the Rydberg states do not depend in practice substantially on the details of the potential near the origin when $n \geq 5$. This means that the features of the trapping coefficient for Rydberg states discussed above are also valid for Rydberg states generated by negative ions. Several authors have reported on experimental evidence for positron trapping at negative ions. ${ }^{12,33}$ For these ions we expect that "gigantic" capture coefficient of the order of $10^{18} \mathrm{~s}^{-1}$ can be observed at low temperatures.

\section{B. Transitions between Rydberg states}

The energy differences between the different Rydberg states are so small that the transitions between them can effectively take place via phonon emission. The transition rates can be estimated from the trapping coefficients for the Rydberg states given above in Fig. 11. For example, the transition rate from the $6 s$ state to the $5 s$ state can be estimated by multiplying the trapping coefficient for the $5 s$ state by the ratio of the volume per atom and the volume corresponding to the extent of the $6 s$ Rydberg state. The average radius $\left(\epsilon n^{2} a_{0}\right)$ for the $6 s$ state is about $420 a_{0}$, which gives a transition rate of $\sim 8 \times 10^{7} \mathrm{~s}^{-1}$. This is a small number when compared to the annihilation rate in semiconductors. The actual calculation gives an even smaller transition rate of $\sim 10^{6} \mathrm{~s}^{-1}$, as Fig. 14 shows. The transition rates in Fig. 14 are between $s$ states at $10 \mathrm{~K}$; the transition rates involving higher $l$ quantum numbers are even smaller because the wave functions for these states are repelled strongly from the origin, i.e., from the center of the vacancy, due to the centrifugal potential. The small rate means that the positron cannot make a transition between two Rydberg states within its lifetime. Thus if the positron trapping process into the ground state at the vacancy uses the Rydberg states as intermediate precursor states, only one 


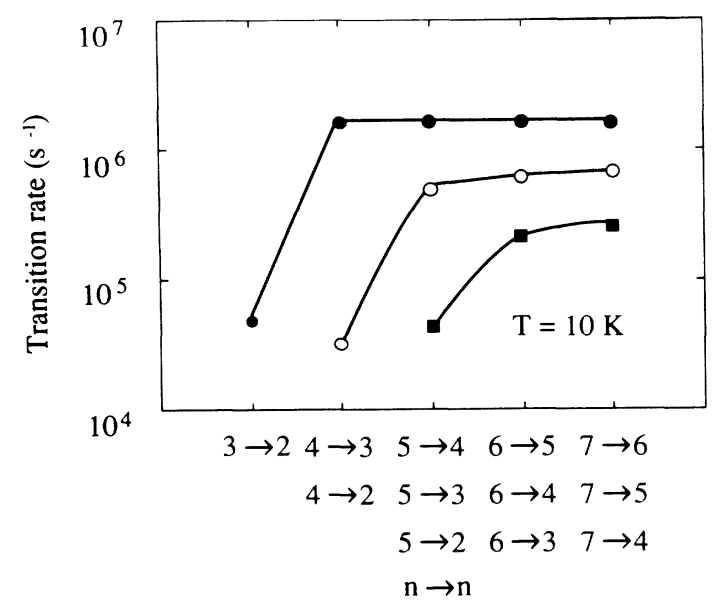

FIG. 14. Transition rates for positron between $s$-type Rydberg states. In the transition the quantum number $n$ changes to $n^{\prime}$ and energy is transferred to a phonon [(iv) in Fig. 1]. The positron states are calculated using the model potential for a singly negative vacancy (Fig. 2). The results correspond to the temperature of $10 \mathrm{~K}$.

Rydberg state can be involved. There is no analog for the Lax cascade ${ }^{30-32}$ suggested for the carrier recombination at charged centers. However, if the second transition is directly to the ground state the energy release is so large that it can effectively occur via electron-hole excitation. In the next subsection, the combined mechanism involving one phonon-mediated step to a Rydberg state and a subsequent electron-hole excitation is discussed in detail.

\section{Transitions from Rydberg states to the ground state}

We have calculated the transition rates for a positron from Rydberg states to the ground state at a negatively charged vacancy by assuming that in the process a localized electron at the vacancy is excited to the conduction band. This transition rate could be again approximated from the values obtained for the direct transitions from the delocalized states to the ground state by the volume scaling. The results from the actual numerical calculations are shown in Fig. 15 in which the transition rates from the $s$ - and $p$-type Rydberg states are given for $n$ varying from 2 to 7 . The transition rate decreases when the quantum number $n$ for the Rydberg state increases reflecting the decrease in the localization of the initial state. For all $s$ states shown and for the $p$ states with small $n$, the transition rates are larger than the bulk annihilation rate in $\mathrm{Si}$ (denoted by the dashed line in Fig. 15). For the states with higher $l$ values the transition rates fall below the bulk annihilation rate. This indicates that while not relevant as precursor states to deep trapping the states with high $l$ values could act at low temperatures as shallow traps for positrons: as discussed in the preceding section also the transition rates between the different Rydberg states are so small that the positron cannot leave this kind of state before annihilation. So far there exists no experimental evidence from this kind of shallow traps.

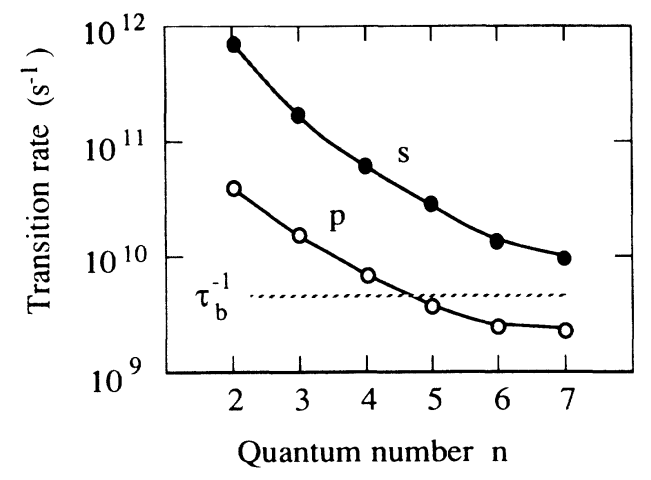

FIG. 15. Transition rates for positron from $s$ - and $p$-type Rydberg states to the ground state as a function of the quantum number $n$. In the transition an electron is excited from a localized state to the conduction band [ $(v)$ in Fig. 1]. Positron and electron states are constructed according to the model potentials of Fig. 2 and parameters in Table I.

\section{Two-state capture mechanism via a Rydberg state}

The above calculations show that at low temperatures the delocalized positron can reach the ground state once it has been trapped into a Rydberg state. The Rydberg state acts as a precursor state. This kind of positron trapping in two stages is analogous to the model presented by Smedskjaer et al. ${ }^{15}$ for positron trapping into jogs or kinks at dislocation lines. In that model the weakly bound positron state delocalized along the dislocation line is the precursor state and the open volume at a point defect serves as the final trap. For a negative vacancy or other vacancy-type defects, the trapping process is a little more complicated because the different Rydberg states act in parallel and in addition direct transition to the ground state exists too. Figure 16 shows the diagram for the trapping process at low temperatures. It is assumed that the annihilation rates for the bulk state and for the weakly localized Rydberg state are equal and denoted as $\lambda_{b}$. The annihilation rate for the ground state at the trap is $\lambda_{t}$. For a given vacancy concentration $c_{v}$ transitions from the delocalized states to the Rydberg state $(n, l)$ take place with the trapping rate $\kappa_{R}$, from the Rydberg

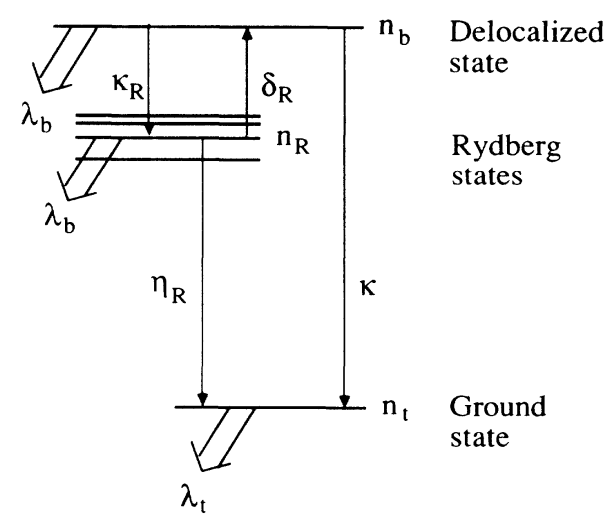

FIG. 16. Schematic view of positron trapping into a negative vacancy in a semiconductor. 
state to the final trap state with the rate $\eta_{R}$, and from the delocalized state directly to the ground state with the trapping rate $\kappa$. The positrons escape due to thermal activation from the weakly bound Rydberg state with the rate $\delta_{R}$ but cannot escape from the ground state. The detrapping rate $\delta_{R}$ and the trapping rate $\kappa_{R}$ into the Rydberg state are related according to Eq. (3). If the rate at which positrons enter the solid is assumed to be constant, the numbers of positrons in different states $\left(n_{b}, n_{r}\right.$, and $n_{t}$ according to Fig. 16) can be solved by applying steady state conditions to the kinetic equations (see Appendix). The detrapping from the Rydberg state leads to a strong decrease in positron trapping as temperature increases. Finally at higher temperatures the direct transition into the ground state takes over.

\section{v. CONCLUSIONS}

We have investigated the various trapping mechanisms operative for positrons in semiconductors. In particular, attention is paid to positron trapping into vacancies with various charge states. The relevant energy release mechanisms are both electronic excitations and phonon emission. The magnitudes and temperature dependencies of the trapping coefficients have been determined in various cases.

In the case of positive vacancies the trapping coefficient depends strongly on the height of the repulsive Coulomb barrier. However, the coefficients obtained with reasonable barrier heights are too low in order that positive vacancies could effectively trap positrons during their lifetime in a semiconductor.

For neutral vacancies the dominant trapping mechanism is found to be electron excitation from a localized state at the vacancy to the conduction band. The trapping coefficient is generally independent of temperature and is about $10^{15} \mathrm{~s}^{-1}$. If there is a scattering resonance near thermal energies, the trapping coefficient can increase rapidly with rising temperature and reach a value of the order of $10^{16} \mathrm{~s}^{-1}$ or even higher at room temperature. The trapping coefficient may reach values as high as $10^{15} \mathrm{~s}^{-1}$ also in electron excitation processes from the conduction band to the valence band, if the host is a narrow-gap semiconductor with electron band masses close to unity.

The trapping coefficients for negative vacancies are at all temperatures larger than those for neutral vacancies. The difference is enhanced at low temperatures, in which negative vacancies show a $T^{-1 / 2}$ divergence. The divergence results because the initial positron wave function behaves like a Coulomb wave, the amplitude of which rises strongly at the vacancy when its energy eigenvalue decreases. This feature is independent of the trapping mechanism in question. At room temperature the dominant capture mechanism is electron excitation from a localized state at the vacancy to the conduction band and the trapping coefficient is of the order of $10^{16} \mathrm{~s}^{-1}$. At low temperatures, trapping into Rydberg states via phonon emission can lead to "gigantic" trapping coefficients of the order of $10^{17}$ to $10^{18} \mathrm{~s}^{-1}$. The Rydberg states act mainly as precursor states to trapping into the ground state. The transition occurs in one step via the electron excitation. There is no multistep, cascade capture process for positrons, because the trapping from one Rydberg state to another is too slow to happen during the positron lifetime. Because detrapping from Rydberg states increases exponentially when temperature rises, the capture via Rydberg states leads to a trapping rate, which decreases strongly as a function of temperature. It may also happen that at low temperatures, when detrapping from the Rydberg states is small, trapping into negative ions effectively competes with trapping into vacancies.

\section{ACKNOWLEDGMENTS}

We have benefitted from several useful discussions with Pekka Hautojärvi, Jari Mäkinen, and Kimmo Saarinen. Furthermore, we acknowledge the important help from Edward Boroński during the early stages of this work, and Jukka Tulkki for his expertise in numerical solving of Coulomb wave functions.

\section{APPENDIX}

In this appendix we derive a relationship between the two-stage capture mechanisms introduced in Sec. IV D and the two-state trapping model used traditionally in analyzing the experimental positron lifetime data. The two-state model is illustrated schematically in Fig. 17. In the model the precursor states are not explicitly treated, but they affect the trapping rate $\kappa^{\prime}$ which is the quantity actually deduced from the measured spectra. We simplify also our two-stage capture mechanism shown in Fig. 16 by assuming that there is only one weakly bound state which describes in the sense of some average all the Rydberg states.

The quantity which can be extracted from the experimental data is the probability that a positron will be trapped and annihilated from the ground state at the defect. For the simplified two-stage trapping mechanism this probability is

$$
\begin{aligned}
A_{t}=\frac{\lambda_{t} n_{t}}{b} & =\frac{\kappa\left(\lambda_{b}+\delta_{R}+\eta_{R}\right)+\eta_{R} \kappa_{R}}{\left(\lambda_{b}+\kappa_{R}+\kappa\right)\left(\lambda_{b}+\eta_{R}\right)+\delta_{R}\left(\lambda_{b}+\kappa\right)} \\
& \approx \frac{\eta_{R} \kappa_{R}}{\left(\lambda_{b}+\kappa_{R}\right) \eta_{R}+\delta_{R} \lambda_{b}}
\end{aligned}
$$

where $b$ is the rate at which positrons enter the solid. The last form above is obtained by approximating $\eta_{R} \gg \lambda_{b}$ and $\kappa<<\lambda_{b}$. The detrapping rate $\delta_{R}$ and the trapping rate $\kappa_{R}$ between the Rydberg state and the delocalized state are related by Eq. (3). In the two-state mod-

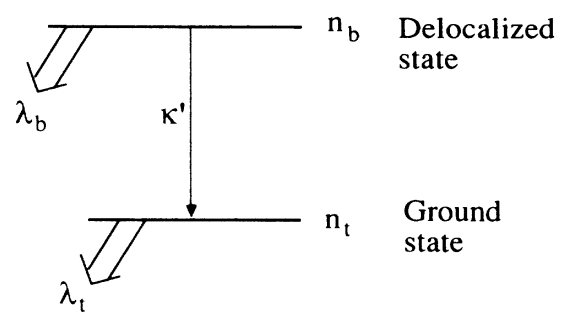

FIG. 17. Two-state positron trapping model. 
el of Fig. 17 the probability for the positron to be trapped and annihilated at the trap state is

$$
A_{t}=\frac{\kappa^{\prime}}{\lambda_{B}+\kappa^{\prime}}
$$

If this probability is required to be equal to that in Eq. (A1) for the precursor model, one obtains for the measured trapping rate the following equation:

$$
\kappa^{\prime}=\frac{\eta_{R} c_{v} \kappa_{R}}{\eta_{R} c_{v}+\kappa_{R}\left(\frac{m_{+} k_{B} T}{2 \pi \hbar^{2}}\right)^{3 / 2} e^{-E_{R} / k_{B} T}} .
$$

Thus at low temperatures the trapping rate $\kappa^{\prime}$ reaches asymptotically $\kappa_{R}$ which is the trapping rate into the Rydberg state. When temperature rises the detrapping from the Rydberg state becomes more and more probable and the trapping rate $\kappa^{\prime}$ starts to fall strongly below $\kappa_{R}$.

Equation (A3) can be used in fitting experimental trapping rates analyzed in the two-state trapping model. There are three independent parameters in Eq. (A3): $\kappa_{R}$, $E_{R}$, and the product $\eta_{R} c_{v}$. If the trapping rate $\kappa_{R}$ is kept as a temperature-independent constant the trapping rate saturates to a constant value at low temperatures. There is evidence for this kind of saturation in the data published by Mäkinen et al. ${ }^{10}$
*Also at Centre d'Etudes Nucléaires de Saclay, Institut National des Sciences et Techniques Nucléaires (INSTN), F-91191 Gif-sur-Yvette CEDEX, France.

${ }^{1}$ S. Dannefaer, Phys. Status Solidi A 102, 481 (1987).

${ }^{2}$ G. Dlubek and R. Krause, Phys. Status Solidi A 102, 443 (1987).

${ }^{3}$ M. J. Puska, O. Jepsen, O. Gunnarsson, and R. M. Nieminen, Phys. Rev. B 34, 2695 (1986).

${ }^{4}$ M. J. Puska and C. Corbel, Phys. Rev. B 38, 9874 (1988).

${ }^{5}$ M. J. Puska, and S. Mäkinen, M. Manninen, and R. M. Nieminen, Phys. Rev. B 39, 7666 (1989).

${ }^{6}$ R. M. Nieminen and M. Manninen, in Positrons in Solids, edited by P. Hautojärvi (Springer-Verlag, Heidelberg, 1979), p. 145.

${ }^{7}$ R. M. Nieminen, in Positron Solid State Physics, edited by W. Brandt and A. Dupasquier (North-Holland, Amsterdam, 1983), p. 359.

${ }^{8}$ S. Dannefaer, S. Kupca, B. G. Hogg, and D. P. Kerr, Phys. Rev. B 22, 6135 (1980).

${ }^{9}$ M. Shimotomai, Y. Ohgino, H. Fukushima, Y. Nagayasu, T. Mihara, K. Inoue, and M. Doyama, in Defects and Radiation Effects in Semiconductors, Inst. Phys. Conf. Ser. No. 59, edited by R. R. Hasiguti (IOP, London, 1981), p. 241.

${ }^{10}$ J. Mäkinen, C. Corbel, P. Hautojärvi, P. Moser, and F. Pierre, Phys. Rev. B 39, 10162 (1989).

${ }^{11}$ P. Mascher, S. Dannefaer, and D. Kerr, in Defects in Semiconductors 15, Materials Science Forum, edited by G. Ferenczi (Trans Tech, Aedermannsdorf, 1989), Vols. 38-41, p. 1157.

${ }^{12}$ K. Saarinen, P. Hautojärvi, A. Vehanen, R. Krause, and G. Dlubek, Phys. Rev. B 39, 5287 (1989).

${ }^{13}$ T. McMullen, J. Phys. F 7, 2041 (1977); 8, 87 (1978).

${ }^{14}$ R. M. Nieminen, J. Laakkonen, P. Hautojärvi, and A. Vehanen, Phys. Rev. B 19, 1397 (1979).
${ }^{15}$ L. C. Smedskjaer, M. Manninen, and M. J. Fluss, J. Phys. F 10, 2237 (1980).

${ }^{16}$ T. McMullen and M. J. Stott, Phys. Rev. B 34, 8985 (1986).

${ }^{17}$ M. J. Puska and M. Manninen, J. Phys. F 17, 2235 (1987).

${ }^{18}$ S. Dannefaer, W. Dean, D. P. Kerr, and B. G. Hogg, Phys. Rev. B 14, 2709 (1976).

${ }^{19}$ M. Stucky, C. Corbel, B. Geffroy, P. Moser, and P. Hautojärvi, in Defects in Semiconductors, Materials Science Forum, edited by H. J. von Bardeleben (Trans Tech, Aedermannsdorf, 1986), Vols. 10-12, p. 265.

${ }^{20} \mathrm{M}$ Jaros, Deep Levels in Semiconductors (Hilger, Bristol, 1982).

${ }^{21}$ B. K. Ridley, Quantum Processes in Semiconductors (Clarendon, Oxford, 1982).

${ }^{22}$ H. H. Jorch, K. G. Lynn, and T. McMullen, Phys. Rev. B 30, 93 (1984).

${ }^{23}$ C. H. Hodges, Phys. Rev. Lett. 25, 284 (1970).

${ }^{24}$ W. Brandt, Appl. Phys. 5, 1 (1974).

${ }^{25}$ M. Manninen and R. M. Nieminen, Appl. Phys. A 26, 93 (1981).

${ }^{26}$ C. O. Rodriquez, S. Brand, and M. Jaros, J. Phys. C 13, L333 (1980).

${ }^{27}$ D. R. Penn, Phys. Rev. 128, 2093 (1962).

${ }^{28}$ R. M. Nieminen and J. Laakkonen, Appl. Phys. 20, 181 (1979).

${ }^{29}$ N. F. Mott and H. S. W. Massey, The Theory of Atomic Collisions, 3rd ed. (Oxford University Press, Oxford, 1987), p. 57.

${ }^{30}$ M. Lax, Phys. Rev. 119, 1502 (1960).

${ }^{31}$ R. A. Brown and S. Rodriquez, Phys. Rev. 153, 890 (1967).

${ }^{32}$ V. N. Abakumov and I. N. Yassievich, Fiz. Tekh. Poluprovodon. 12, 3 (1978) [Sov. Phys. - Semicond. 12, 1 (1978)].

${ }^{33}$ C. Corbel, F. Pierre, P. Hautojärvi, K. Saarinen, and P. Moser, Phys. Rev. B (to be published). 